\title{
Atomic scale modelling of the cores of dislocations in complex materials part 1: methodology $\dagger$
}

\author{
Andrew M. Walker, $\ddagger^{* a b}$ Julian D. Gale, $^{c}$ Ben Slater $^{a}$ and Kate Wright ${ }^{a b c d}$ \\ ${ }^{a}$ Davy Faraday Research Laboratory, The Royal Institution of Great Britain, 21 Albemarle \\ Street, London, UKW1S 4BS.E-mail: andrew.m.walker@anu.edu.au; \\ Fax: +61 (0)26125 8253; Tel: +61 (0)261254076 \\ ${ }^{b}$ Department of Earth Sciences, University College London, Gower Street, London, \\ UK WC1E $6 B T$ \\ ${ }^{c}$ Nanochemistry Research Institute, Department of Applied Chemistry, Curtin University of \\ Technology, P.O. Box U1987, Perth, 6845, Western Australia \\ ${ }^{d}$ Department of Chemistry, Christopher Ingold Laboratories, University College London, \\ 20 Gordon Street, London, UK WC1H OAJ
}

Received 22nd April 2005, Accepted 6th July 2005

First published as an Advance Article on the web 3rd August 2005

Dislocations influence many properties of crystalline solids, including plastic deformation, growth and dissolution, diffusion and the formation of polytypes. Some of these processes can be described using continuum methods but this approach fails when a description of the structure of the core is required. To progress in these types of problems, an atomic scale model is essential. So far, atomic scale modelling of the cores of dislocations has been limited to systems with rather simple crystal structures. In this article, we describe modifications to current methodology, which have been used for strongly ionic materials with simple structures. These modifications permit the study of dislocation cores in more structurally complex materials.

\section{Introduction}

For more than three decades, computer simulation methods have been used to either complement experiment, or to overcome experimental limitations in the study of the atomic-scale detail of line defect cores in crystalline solids. However, these studies have so far been limited to crystals with very simple structures; namely alkali halides and binary oxides with the rock salt structure, various metals and some semi-conductors. In this article we describe developments that enable one to study the cores of screw and edge dislocations in much more complex ionic and semi-ionic materials. The ability to model the fine structure of dislocation cores in these materials should open up diverse new avenues of research. For example the plastic deformation of crystalline solids is often controlled by the glide and climb of edge dislocations, ${ }^{2,3}$ two processes which are directly controlled by the structure of the dislocation core. Crystal growth is often enhanced by the emergence of screw dislocations at the crystal surface; a process that results in the formation of characteristic growth spirals and, in some materials, can lead to interesting long period polytypism. ${ }^{4}$ The presence of line defects in crystals can also open pathways for the rapid diffusion of point defects - with consequences for plasticity. ${ }^{5}$ One of most visually striking consequences of dislocation mediated growth is that the external crystal morphology or habit can be dramatically affected. For example, natural diamonds are typically bipyramidal but under conditions of low supersaturation, where spiral growth dominates, cubic forms are seen. ${ }^{6}$ A more challenging technological problem in crystal engineering is controlling the morphology of pharmaceuticals, where morphology is a patentable property. Evidently, an

$\dagger$ For part 2 see ref. 1.

$\ddagger$ Current address: Research School of Earth Sciences, Australian National University, Mills Road, Canberra, ACT 0200, Australia. extensive study into the structure and dynamical behaviour of this class of defects is required for a variety of materials.

The major problem associated with simulating dislocations is that all of the atoms in the crystal containing the dislocation are significantly displaced from their location in the perfect crystal by the dislocation, and the strain energy associated with the dislocation decays only logarithmically with distance from the dislocation line. ${ }^{3}$ The modelling techniques used to study point defects and surfaces, where the atomic structure at long range from the defect is only slightly perturbed from or is taken to be identical to the structure of the perfect crystal, are therefore not immediately available. There are two approaches to overcome this problem; the first utilises a similar method to that employed to study point defects or surfaces, where an atomistic model of the defect is embedded into a more approximate model of the crystal far away from the defect. The second approach is to impose an artificial periodicity on the simulation model by placing a number of dislocations within a simulation supercell. This makes it possible to perform the calculation using any computer code designed to model perfect crystals (using periodic boundary conditions) at the atomic scale at the expense of having to deal with dislocation-dislocation interactions. (Such an approach is particularly useful if density functional theory with a plane-wave rather than local orbital basis set is to be used.)

In order to use periodic simulation cells, special cell geometries must be constructed, in which the net force on the core of the dislocation from its periodic images is cancelled. ${ }^{7,8}$ This approach allows for the use of standard plane-wave codes for the simulation of dislocations in metals, such as molybdenum and tantalum, ${ }^{9}$ and in semiconductors where the suppression of dislocations is vital for computer chip manufacture. Studies of the structure and electronic properties of dislocations in semiconductors include work on edge $\mathrm{e}^{7,10}$ and screw ${ }^{8}$ dislocations in silicon, studies of an extended defect structure in 
layered $\mathrm{Si} / \mathrm{Ge}$ crystals, ${ }^{11}$ and the incorporation of hydrogen ${ }^{12}$ and other impurities ${ }^{13}$ at edge dislocations. Comparisons of the structure of dislocations in silicon and carbon (diamond) have also been made. ${ }^{12,14} \mathrm{~A}$ limitation of using periodic boundary conditions is that the energy of the simulation cell with respect to a reference ideal crystal includes terms for dislocationdislocation interactions analogous to defect-defect interactions found in supercell studies of point defects. ${ }^{15}$ While these can be calculated and corrected for, ${ }^{8}$ it is not at all clear that this technique is tenable when applied to ionic systems. This is because in addition to the elastic interactions, there will inevitably be electrostatic interactions between dislocation cores, which are expected to develop dipoles and higher multipoles due to the rearrangement of their ions. In addition, point defects, jogs and kinks on the dislocation line can carry charge and thus interact over larger distances.

Some of the earliest work modelling dislocations, dating from the early 1970's, was performed on metals and utilised the "embedded" approach. An example is the work on $\alpha$-iron ${ }^{16-18}$ which also provided a convenient test bed for new methodologies. These studies used a similar approach to that described in this article, with a straight dislocation in a simulation cell with 1-D periodicity and a two-region approach to the calculation of dislocation energy. More recently, advances in interatomic potentials for metallic systems coupled with new flexible boundary conditions (see below) have led to work on technologically important metals. These include studies of dislocations in tantalum, ${ }^{19}$ iron, ${ }^{20}$ aluminium ${ }^{21,22}$ and molybdenum. ${ }^{22}$ Many of the recent atomic simulations of dislocations in metals have been focused on linking an atomic scale model of the dislocation core with a "bottom up" mesoscopic description of plasticity and strength. ${ }^{23}$ Pertinent examples are the simulation of cross slip in copper ${ }^{24}$ and recent simulations of very large systems of iron, ${ }^{25}$ which suggest a new picture of plasticity controlled by the cross slip of screw dislocations.

At the time of the earliest studies in the 1970's interatomic potential models for metals were poorly developed. In contrast, potential models for simple ionic materials were well developed and generally reliable. Much of the early work was therefore performed on this class of materials. ${ }^{26,27}$ Of particular note is the work undertaken by Puls and co-workers who developed the PDINT code for the simulation of dislocations in cubic ionic materials. Concentrating on $\mathrm{MgO}$ as a model system this group first used a simple shell model with rigid boundary conditions to calculate the Peierls energy for the glide of the $(a / 2)\left[110\right.$ ] edge dislocation. ${ }^{26,28}$ A breathing shell model was then used with the Flex-II boundary conditions to recalculate the geometry of the dislocation core ${ }^{29}$ and to re-evaluate the Peierls energy barrier. ${ }^{30}$ The code was then developed further in order to model point defect-line defect interactions, again in $\mathrm{MgO} .{ }^{31}$ Further work involved comparisons between the behaviour of dislocations in $\mathrm{MgO}, \mathrm{NaCl}$ and $\mathrm{NiO} .{ }^{32}$ Compared to studies of metals and semiconductors, recent interest in ionic systems has been rather limited ${ }^{33}$ with the notable exception of Watson and co-workers. ${ }^{34-36}$ This group used the METADISE code $^{34}$ to study screw dislocations in $\mathrm{MgO}^{35}$ and the effect of these dislocations on the $\mathrm{MgO}[100]$ surface. ${ }^{36}$ The work of Sayle is also worth highlighting, ${ }^{37}$ in this study one oxide is "deposited" on to the surface of another on the computer and, in some cases, edge dislocations are seen to form spontaneously in order to relieve strain caused by mismatch in the lattice parameters of the two materials.

Despite this large body of work, there appears to have been no attempt to model dislocations in structurally complex low symmetry materials, with many ions in the crystal basis. This is perhaps surprising given the importance that dislocations must play in the behaviour of these materials and the regular application of computational models to understand the point defect and surface properties of complex systems. The major problems to be overcome are that (1) the systems are generally of low symmetry and so anisotropic elasticity must be used and (2) any arbitrary simulation cell with 1-D periodicity is likely to have a net charge or macroscopic dipole across it. A third issue relates to the method of calculating the Coulomb contribution to the lattice energy, the atomistic model of the dislocation is typically sizeable $(\sim 5 \mathrm{~nm})$ and sophisticated charge summation schemes are necessary to evaluate the electrostatic energy with accuracy in one dimension, which adds both complexity and computational expense to the calculation. ${ }^{38,39}$ In the following sections of this paper, we outline a methodology to address these issues and discuss the analysis of the structure of dislocation cores. The advances described permit the modelling of dislocations in complex materials that, thus far, have not been amenable to study. We examine some of the possibilities offered by this approach in a companion paper where the structures of the cores of screw dislocations in crystalline materials ranging from ceramics to a pharmaceutical are described.

\section{Methodology}

We base our methodology on the many earlier studies utilising $1 \mathrm{D}$ periodicity, reviewed in the introduction. The task is to find the structure of the core of edge and screw dislocations, and the energy cost associated with introducing them into an otherwise perfect ionic crystal. In general, this problem is complicated by the fact that dislocations can be curved (i.e. the dislocation line is not straight), they may bifurcate, they may be moving and their Burgers vector can vary with position along the dislocation line. The dislocation model adopted here simplifies this situation by constraining the dislocation line to be infinite, straight and stationary with a constant Burgers vector. This allows the dislocation to be modelled in a cell that is periodic in one dimension (along the dislocation line). Perpendicular to the dislocation line the modelled crystal is considered infinite and non-periodic. However, by considering the part of the crystal a long distance from the dislocation line to be under relatively small strain and using methods appropriate to the description of an elastic continuum, a two region approach to the calculation of dislocation formation energy can be constructed. The structure of the core can then be found with a relatively small number of atoms in the simulation cell. Of course, this situation is a simplification of reality where dislocations must form a closed loop, emanate from another lattice defect or terminate at the edge of the crystal.

The methodology is described in detail below; first the methods used to set up the simulation cell for edge and screw dislocations are described, this is followed by details of the actual simulation (which amounts to an energy minimisation problem, in this case performed using the latest revision of the GULP $\operatorname{code}^{40}$ ). Finally, techniques used to analyse the properties of the resulting defect structure are considered.

Our emphasis in this publication is to consider the problems associated with studies of dislocations in complex ionic materials. We therefore only consider the simple case of fixed or rigid boundary conditions to represent the crystal away from the core within the simulation. More sophisticated approaches, such as Flex-S, ${ }^{17}$ Flex-I, ${ }^{18}$ Flex-II ${ }^{16,41}$ or the more modern domain decomposed approach of Rao et al., ${ }^{20}$ could be used, but we reserve their implementation in the ionic case for future study. The approximations inherent in the use of rigid boundary conditions are described below.

\section{The simulation cell}

The first task is to construct a correctly orientated atomistic simulation cell of the appropriate dimensions containing a dislocation. The cell is chosen to have a circular cross section approximately centred on the origin of the dislocation. The convention used through out this work is for the cell to be periodic along the Cartesian $z$-axis and non-periodic along on the 
other two orthogonal axes ( $x$ and $y$ ) where it is to be embedded into an elastic model of the infinite crystal. The dislocation line therefore lies along $z$. Screw dislocations must then have a Burgers vector parallel to $z$ and edge dislocations have a Burgers vector in the $x y$-plane. Dislocations with a general mixed character therefore have components of the Burgers vector in all three directions. The exact orientation of the cell with respect to the cell parameters is chosen to simplify the introduction of the dislocation as described below. ${ }^{42,43}$ In cases where the crystal, as described by the chosen set of interatomic potentials, is elastically anisotropic, it is necessary to calculate the elastic compliance tensor $\left(s_{l m}\right)$ for the bulk material expressed in the Cartesian frame of reference described above.

An additional complication when modelling ionic systems is that the cell must be charge neutral to ensure that the total energy is finite. It is also desirable to minimise the total dipole perpendicular to the dislocation line (the dipole component along the periodic repeat is undefined as its value is dependent on an arbitrary choice of origin). Any dipole would be screened out in real crystals (e.g. by point defects, other dislocations or surface effects) and a large dipole across the simulation cell may result in artificial forces on the atoms in the model. We have developed several approaches that assist in achieving a charge neutral, low dipole cell and three of these are illustrated in the examples given in the accompanying publication. Briefly, if the crystal consists of strings of atoms with no net charge parallel to the dislocation line (as in $\mathrm{MgO}$ where $\cdots \mathrm{Mg}-\mathrm{O}-$ $\mathrm{Mg}-\mathrm{O} \cdots$ strings can be found) then any cut will achieve charge neutrality and one only has to place the centre of the circular cell on a rotation axis to minimise the dipole. In the general case, neutral strings do not occur and one solution is to build the cell out of whole molecules. This results in a neutral cell and, if a rotation centre is selected for the cell origin and the dipole on the molecules is small, then the total dipole across the cell will be small. Using this method, the profile of the cell is no longer circular, but instead it has somewhat ragged edges. The third approach is to build the cell out of "charge neutral units" in a manner exactly analogous in one dimension to the zero dimensional approach used by Braithwaite et al. ${ }^{44}$ in their study of point defects in forsterite. This method is explored in more detail in the accompanying publication and we have previously used it for our model of screw dislocations in zeolite A. ${ }^{45}$

Once the bulk-like cell is generated, the second step is to introduce a dislocation into it. In order to achieve this, it is temporarily assumed that the crystal is an anisotropic, homogeneous, infinite, linear elastic body with elastic constants previously determined using the atomistic model. The atoms are considered to be embedded within this elastic body; they will move with it and have no effect on its behaviour. As the dislocation is introduced into this body, the atoms are passively carried from their location in the un-dislocated bulk cell into their location in the dislocated crystal and this new configuration is used as the starting point for an atomic simulation of the core. The atomic simulation, which is performed after the dislocation is introduced, lifts the assumption of linear elasticity and corrects the inaccuracies introduced by such an approximation. Thus, the problem of introducing the dislocation is thus reduced to introducing a dislocation into an elastic body and keeping track of where particular pieces of the body (representing the atoms) move as the dislocation is introduced.

Introducing the dislocation into the elastic body corresponds to the following process: ${ }^{3,4}$ (1) a small amount of material from the centre of the cell is removed, (2) a cut is made from the edge of the cell to the hole in the centre, (3) the two sides of the cut are displaced by the Burgers vector such that the two sides remain parallel, (4) the cut is cemented together and the system is allowed to reach elastic equilibrium. This procedure is shown diagrammatically in Fig. 1. When an elastic body undergoes this process the displacement of any piece of the body is known as its elastic displacement and, the displacement of all atomic consti-
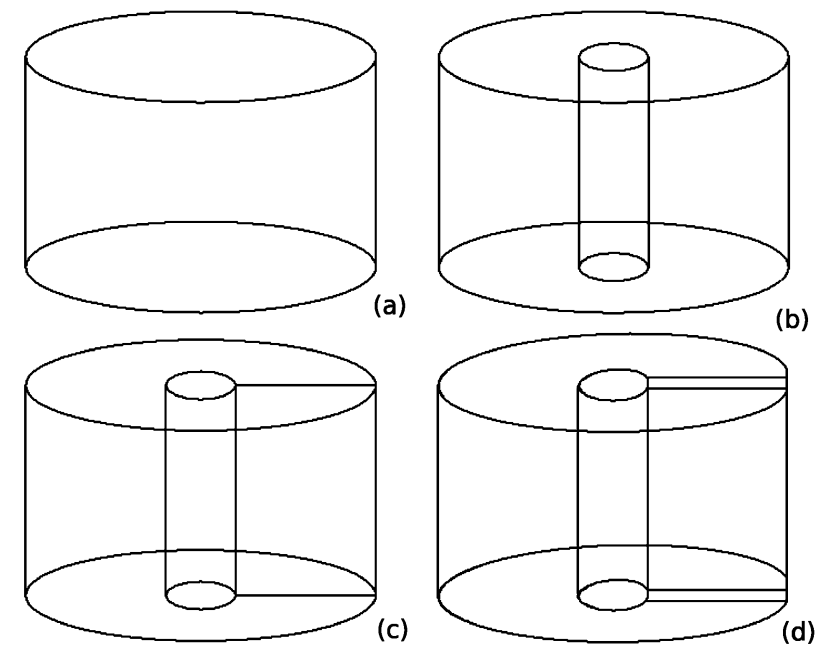

Fig. 1 Schematic diagram showing the process used to introduce a screw dislocation into a perfect crystal by Volterra's procedure. The diagram shows a screw dislocation but an equivalent process occurs for an edge dislocation. (a) represents the bulk-like cell generated by multiplication of the crystal unit cell, in (b) a portion of the crystal is removed, in (c) a cut is made and in (d) the two surfaces are moved past each other. In order to introduce an edge dislocation the movement in (d) would be at right angles to that shown. Volterra's linear elastic displacement field maps points in (a) onto the equivalent point in (d) if the hole in the centre of the crystal is sufficiently large and there is no traction on its walls.

tuent parts of the body are described by the elastic displacement field. For an elastic body with low symmetry this field must be found numerically, however in some cases symmetry permits analytical solutions for the displacement field (see the Appendix). The displacement field can be viewed as a mathematical function that maps the location of a point in the bulk cell to the equivalent point in the dislocated cell as if the "cut and slip" process described above had been performed. In practice, this field is evaluated for the position of each atom in the bulk like cell, and the displacement is applied to the atom in question. The result is the introduction of a dislocation into the cell. It should be noted that the solution to the linear elastic displacement field of the dislocation and is only a function of the Burgers vector, the orientation of the dislocation, and the elastic properties of the crystal. Consequently, good reproduction of the bulk elastic constants is a critical prerequisite for a model of a dislocation. The calculated elastic displacements are exact a large distance from the core (where the strain is infinitesimal), but are only approximate close to the core. Subsequent energy minimisation, with atoms again explicitly considered, corrects the geometry of the core and its surroundings.

\section{Finding the geometry of the dislocation core}

The method described in the previous section allows the generation of a simulation cell containing a dislocation. However, all the expressions used to introduce the dislocation given in the Appendix assume that the displacements of the atoms in the simulation cell can be described by linear elastic theory (the hole in Fig. 1 is, in practice, made infinitesimally small). This results in four causes of error in the location of the atoms in the starting cell that mean that the starting configuration is not at an energy minimum. The geometry is corrected by energy minimisation using an atomistic model and the difference between the positions of the atoms found by the elastic and atomistic calculation (i.e. the displacements of the atoms during the energy minimisation) is termed the core displacement field. This is described below, and its typical form is illustrated in Fig. 2. The presence of this field arises from the following four errors in the elastic method used in setting up the initial simulation geometry: 


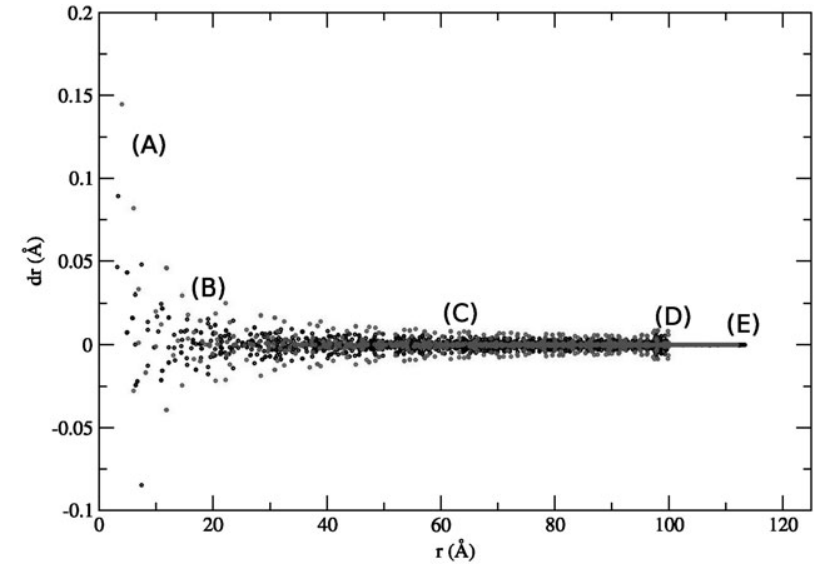

Fig. 2 Typical features of the "core" displacement field (the difference between the displacements calculated using linear elasticity and the results of the atomistic model) as a function of radius, $r$. This figure shows the radial component, $\mathrm{d} r$, for a screw dislocation in zeolite A, examples for other systems are given in the supplementary information to the accompanying paper. From the core at $r=0 \AA$ to the edge of the model at $r=115 \AA$ the following general features are observed: (A) Large displacements at the core due to rearrangement of the core structure (caused by error 3). (B) Decaying displacements caused by non-linear elastic effects and the dissipation of forces generated by the core rearrangement (caused by errors 1 and 4). (C) Eventual near constant pattern of displacements due to the rearrangement of atoms in strained cells (caused by error 2). (D) Region 1-region 2 interface effect between homogeneously strained region 2 and free atomistic region (caused by error 2). (E) No movement in region 2 .

1. The displacements are taken to correspond to linear elastic theory; this is equivalent to assuming that the strains (i.e. the relative displacement of the atoms to one another) are small. This assumption is clearly invalid close to the dislocation origin where the strains can be very large. In a macroscopic sense, these non-linear terms manifest themselves in a change in the elastic constants with strain and, in general, they result in patterns of deformation not expected from a consideration of only the linear terms. For example, in a non-linear isotropic elastic body with five independent elastic constants the introduction of a screw dislocation causes displacement of material outwards, away from the dislocation origin. This pattern of displacement is completely absent from the equivalent linear isotropic solution. ${ }^{46}$

2. The strain of each crystal unit cell around the dislocation is finite and can be quite large. In general large strains of a unit cell result in relative displacement of the ions within it (except in cases where the atoms cannot move and preserve the cell's symmetry). If the crystal is made up of covalently bonded molecules one would expect the bonds within the molecules to be relatively unstrained while most of the strain of the cell is taken up in changing the molecules' separation and orientation. The methodology outlined here results in all atomic separations being treated in the same way; the strain in the cell is homogeneous. The approximation introduces a discontinuity between the fixed and relaxed regions, but tests for finite size effects show that this error is localised and does not affect the structure of the dislocation core ${ }^{45}$ Indeed this discontinuity can be observed in the core displacement field and is seen to decay quickly (see Fig. 2), as would be expected for what is effectively an interfacial effect.

3. The structure of the core is, by definition, not known from elastic theory. The elastic description of the dislocation has a singularity in energy at $r=0$, and the atomic displacements are discontinuous. The method assumes that the structure of the core can be found from the starting approximation given by linear elastic theory.

4. Reconstruction of the atomic structure of the dislocation core may well be accompanied by a dilation, contraction or twisting of the core. This in turn applies forces from the core onto the surrounding crystal, which responds elastically. This effect is obviously not accounted for in setting up the cell.
In general, several solutions are available to the problem that linear elasticity is inaccurate close to the dislocation core; if the structure of the core itself is not a concern, but its effect is, then non-linear elastic theory can be applied. ${ }^{46}$ However, if the structure of the core is of interest, then its constituent atoms must be explicitly treated. This is a particular problem since the geometry of the core depends on the position of the atoms surrounding the core and the position of the atoms around the core depends in turn on the geometry of the core. There is a solution to this problem: the position of atoms a long distance from the core can be found using elastic methods given only a knowledge of the elastic constants, the direction of the dislocation line and the Burgers vector. If enough atoms can be simulated such that the non-linear elastic terms (associated with the large strains near to the core) and the core dependent effect decays before the edge of the free atomistic region is reached, then so called fixed boundary conditions can be used. In such a simulation, a large portion of the crystal around the dislocation core is relaxed to minimise the energy of the whole cell, while the atoms near the edge of the simulation cell are held fixed at the positions determined by linear elasticity. This is the approach used in the present work. A check that the size of the relaxed portion of the crystal is large enough to accommodate the nonlinear and core effects is to calculate the energy derivatives on the innermost atoms that are held fixed during the relaxation. If these values are small then the free region is large enough. In cases where insufficient numbers of atoms can be included in the simulation to meet this condition, then one of the flexible boundary conditions ${ }^{16-18,20}$ can be used.

The methodology described so far is quite general and does not depend on the form of the interatomic potential used. However, in order to model ionic, or partially ionic, systems the cell described above must contain charged ions, which interact via a Coulombic potential. In one dimension the standard Ewald summation is not available but, in principle, this is not a problem because in one dimension (in contrast with the two or three dimensional cases) the Coulomb summation is absolutely, if slowly, convergent. The slow convergence makes a simple real space summation inappropriate for these terms. However, several methods are now available which make the problem tractable. In this work, the Coulomb summations of both Saunders et al. ${ }^{39}$ and of Wolf et al. ${ }^{38}$ are used. Both have been implemented in the latest revision to the GULP code ${ }^{40}$ where we have found that the optimal choice of summation method depends on the size of cell required.

For cells with a smaller radius, the summation of Saunders et al., ${ }^{27}$ originally developed for the simulation of polymers is preferred. This summation is superior to that used by, for example, Hoagland et al. ${ }^{27}$ or Puls and Norgett. ${ }^{26}$ Firstly, because the new approach does not require strings of atoms with a net charge of zero parallel to $z$, and secondly because Watson et al. ${ }^{35}$ report that the new summation exhibited faster convergence. The key to enhanced convergence lies in application of a neutralising background for each atom in the system and the use of the Euler-MacLaurin summation formula. Using this approach the Coulomb energy is given by: ${ }^{40}$

$$
\begin{aligned}
U_{\text {Coulomb }}= & \frac{1}{2} \sum_{m=-M}^{+M} \sum_{i=1}^{N} \sum_{j=1}^{N} \frac{q_{i} q_{j}}{r_{i j}+m a}-\frac{1}{2} \sum_{i=1}^{N} \sum_{j=1}^{N} \frac{q_{i} q_{j}}{a} \\
& \times\left[\ln \left(\sqrt{(u+z)^{2}+x^{2}+y^{2}}+u+z\right)\right. \\
& \left.+\ln \left(\sqrt{(u+z)^{2}+x^{2}+y^{2}}+u-z\right)-2 \ln a\right] \\
& +\frac{1}{2} \sum_{i=1}^{N} \sum_{j=1}^{N} q_{i} q_{j}\left[\xi\left(M, r_{i j}\right)+\xi\left(M,-r_{i j}\right)\right],
\end{aligned}
$$


where the sums are over all images of $N$ atoms separated by a distance $r$ and with charge $q$ in each of $M$ unit cells which have a periodic repeat, $a$, in the $z$ direction. Components of the atomic separation is given by $x, y$ and $z$, and the other variables are defined by:

$$
\begin{gathered}
u=a\left(M+\frac{1}{2}\right) \\
\xi\left(M, r_{i j}\right)=-\sum_{i=1}^{M} E_{i} a^{2 i-1}\left(\frac{\partial}{\partial u}\right)^{2 i-1}\left((u+z)^{2}+x^{2}+y^{2}\right)^{-1 / 2}
\end{gathered}
$$

and $E_{i}$ are coefficients of an Euler-MacLaurin series, with the first five terms given by: $\frac{-0.5}{12}, \frac{0.875}{720}, \frac{-0.96875}{30240}, \frac{0.9921875}{1209600}, \frac{-0.998046875}{47900160}$. The accuracy of the total Coulomb energy depends on the value chosen for $M$, in practice the energy is evaluated for increasing values of $M$ until the energy converges to a suitable accuracy, comparable to that yielded by the Ewald method. In practice, the sum is found to converge rapidly, with $M \leq 5$.

As the cell radius increases, the Saunders summation becomes increasingly inefficient. A better approach for these large cells is the real-space Wolf summation. ${ }^{38}$ This has the advantage of scaling linearly with number of atoms in the simulation cell. However, the potential model should be fitted taking account of the fact that the Wolf summation is to be used (i.e. during fitting the Coulomb contribution to the energy and energy derivatives should be evaluated using the Wolf sum). The summation scheme is based around the concept of ensuring that the sum of the charges of all ions within a spherical cut-off region of radius $R_{\text {cut }}$ is equal to zero and that the potential goes smoothly to zero at the cut-off. This is implemented by placing an image of every atom within the cut-off sphere on the edge of the sphere projected through the central atom. Convergence is enhanced by using a damped form of the potential equivalent to the real space part of the Ewald sum. The amount of damping is determined controlled by a convergence parameter, $\alpha$. In this form the Coulomb energy is written:

$$
\begin{aligned}
r_{i j}<R_{\mathrm{cut}}, U_{\text {Coulomb }}= & \frac{1}{2} \sum_{i} \sum_{j} q_{i} q_{j} \\
& \times\left(\frac{\operatorname{erfc}\left(\alpha r_{i j}\right)}{r_{i j}}-\lim _{r_{i j} \rightarrow R_{\mathrm{cut}}}\left\{\frac{\operatorname{erfc}\left(\alpha r_{i j}\right)}{r_{i j}}\right\}\right) \\
& -\sum_{i} q_{i}^{2}\left\{\frac{\operatorname{erfc}\left(\alpha R_{\mathrm{cut}}\right)}{2 R_{\mathrm{cut}}}+\frac{\alpha}{\pi^{1 / 2}}\right\}
\end{aligned}
$$$$
r_{i j} \geq R_{\text {cut }}, U_{\text {Coulomb }}=0
$$

where the final term is the subtraction of the self energy of the smoothing Gaussian.

On a related note, the Madelung potential should be correct for all the atoms that are allowed to move during the simulation. This can easily be achieved by making the shell of atoms embedded in the elastic continuum thick enough to achieve convergence in the dislocation energy. Note that if the summation of Wolf et al. ${ }^{38}$ is used this condition can always be met by setting the shell to be thicker than the real space cut-off radius.

In short, the simulation itself involves taking a cell containing a dislocation with the atoms near the centre of the cell positioned according to an elastic approximation. Generally, this atomic arrangement does not correspond to the equilibrium geometry of the core and this deficiency is remedied by fixing the atoms around the edge of the cell (which are correctly positioned) and minimising the energy of the cell.

\section{Analysis of the dislocation structure}

Once the geometry of the dislocation core and surrounding crystal has been established, it is possible to describe the dislocation in several ways. In particular, the displacement field of the dislocation is of relevance. This is defined as the change in position of the atoms in the relaxed cell compared to those in the bulk crystal (or the bulk-like 1D cell). This vector field can be split into two components, the first is the linear elastic displacement field, which is already known; it is analytically determined before starting the simulation (see the Appendix). The second component is the deviation of the linear elastic displacement field from the total displacement field caused by the four processes outlined above. This part will be termed the core displacement field (although not all contributions to it are strictly due to the presence of the core). There are equivalent stress and strain fields. Also of interest is the actual structure of the core itself. In some cases the structure is similar to the structure of the non-defective crystal, but in others significant deformation and reconstruction is observed.

The total energy of the simulation cell is not a particularly useful quantity in isolation. This is because this energy depends on the size of both the free and fixed portions of the simulation cell as well as on the exact method used to terminate the simulation cell. In addition, the accuracy of the total energy of the cell will crucially depend on the ability of any potential model to describe 'non-equilibrium' regions of the potential energy surface. However, a measure of the thermodynamic stability of the dislocation is the "dislocation formation energy", defined as the work done (per unit length of dislocation) by introducing the dislocation into an otherwise perfect crystal. ${ }^{3}$ Expressing this is not a simple matter, as this energy does not converge with the size of the simulation cell (in contrast with models of point or planar defects). A quantity that can be expressed is the dislocation energy stored within a certain radius of the dislocation origin. The basic theory of dislocations splits this energy into two components, ${ }^{3}$ the first stored within the dislocation core and the second stored by the elastic response of the rest of the crystal. As an example, in an elastically isotropic material the energy stored by a screw dislocation within a cylinder of radius $r$ centred on the dislocation line is described by:

$$
E(r)=E(\text { core })+\frac{G b^{2}}{4 \pi} \ln \left(\frac{r}{r_{0}}\right) .
$$

$E$ (core) is the energy stored within the core which has radius $r_{0}$, $b$ is the length of the Burgers vector and $G$ is the shear modulus. Similar expressions exist for anisotropic crystals replacing $G$ with another combination of the elastic constants. This "energy factor", which depends on the symmetry of the elastic constant tensor (and therefore the point group of the crystal) in a similar manner to the elastic displacement field, has been derived for a number of point groups by Steeds. ${ }^{43}$

The dislocation energy is also the energy difference between the simulation cell with and without the dislocation, stored within a given radius and this definition is used to extract dislocation energies from our models, i.e. $E(r)$ is given by:

$$
E(r)=E_{\mathrm{d}}(r)-E_{\mathrm{p}}(r)
$$

Where $E_{\mathrm{d}}(r)$ and $E_{\mathrm{p}}(r)$ are the energy stored with radius $r$ in the dislocated and perfect cell respectively. $E_{\mathrm{d}}(r)$ and $E_{\mathrm{p}}(r)$ can be evaluated for values of $r$ directly for any value of $r$ smaller than the radius of the model by dividing the simulation cell into two parts (Fig. 3). Region 1, centred on the origin of the dislocation, is cylindrical with a radius $r$, region 2 is the rest of the cell. The total energy of the cell can now be split into four terms:

$$
E_{\text {total }}=E_{11}+E_{12}+E_{21}+E_{22},
$$

where $E_{11}$ is the total interaction energy (including both short range and Coulomb contributions) between all pairs of ions in region $1, E_{12}$ is the interaction energy between atoms in region 1 and atoms in region 2, $E_{21}$ is the interaction energy of atoms in region 2 with atoms in region 1 and is equal to $E_{12}$, and $E_{22}$ is the interaction between pairs of atoms within region 2. $E_{\mathrm{d}}(r)$ is 


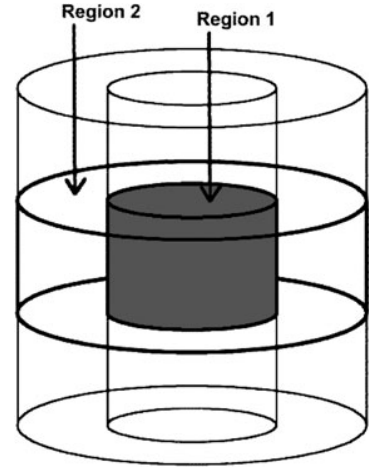

Fig. 3 Cell setup for the evaluation of $E(r)$. The radius of region 1 is chosen to be equal to $r$, region 2 must then be sufficiently large to correctly yield region 1-region 2 interaction energies. This limits the maximum value of $r$ that can be used to evaluate $E(r)$ for a particular simulation cell.

then given by:

$$
E_{\mathrm{d}}(r)=E_{11}+E_{12},
$$

and $E_{\mathrm{p}}(r)$ can be found in the same way. This means that many values of $E(r)$ can be found and these used to fit to eqn. (3) and the core radius and energy can then be extracted. A further problem arises if, for any of the selected values of $r$, region 1 has a net charge (balanced by an equal and opposite charge on region 2). Such a situation is inevitable in low symmetry crystals and the solution is to calculate $E_{\mathrm{p}}(r)$ in an alternative way. Using a parameterised model the lattice energy of the bulk material is usually defined:

$$
E_{\text {lattice }}=\sum_{i} U_{i}+\frac{1}{2} \sum_{i j} U_{i j}+\ldots
$$

but, as long as the atoms do not move, the total lattice energy can be redefined as the sum of the binding energies of all the atoms in the cell:

$$
E_{\text {lattice }}=\sum_{i} E_{\text {binding }}=\sum_{i}\left(U_{i}+\frac{1}{2} U_{i j}+\ldots\right)
$$

and these energies can be found for each symmetrically distinct atom in the unit cell. The solution to the charge problem is then to sum up $E_{\mathrm{p}}(r)$ for all atoms in region 1 of the defective cell and use this value in eqn. (4).

\section{Summary}

We have described an approach to modelling screw and edge dislocations in structurally complex and diverse materials. The major developmental advances associated with the approach described here include (i) the application of anisotropic elastic theory, (ii) efficient methods of calculating the Coulomb contribution to the total energy and (iii) a charge-neutral group method of terminating the simulation cell that guarantees charge neutral cells. The combined use of the elastic and atomistic methods described here leads to a description of the core structure of dislocations via a computationally expedient route. In an accompanying paper, we examine the cores of screw dislocations in a number of materials ranging from the ionic, to the semi-ionic and the purely covalent and demonstrate the utility of the methodology outlined above.

\section{Appendix: The linear elastic displacement field around a dislocation}

In order to set up the dislocation models described in this paper the linear elastic displacement field must be known. This appendix gives the equations for the field for screw and edge dislocations in isotropic and some anisotropic crystals.

Assuming linear elasticity, and neglecting core and surface effects, the displacement field around a dislocation in an elastically isotropic material is well known; Volterra provided a general, analytical solution to the displacement field needed to introduce the dislocation into an elastically isotropic material. ${ }^{4}$ In the case of a pure screw dislocation, with a Burgers vector of length $b$, and in the frame of reference described above, the expression is straight forward: ${ }^{3}$

$$
\begin{aligned}
& u_{x}=u_{y}=0, \\
& u_{z}=\frac{b}{2 \pi} \arctan \frac{y}{x} .
\end{aligned}
$$

Any point $(x, y)$ simply moves parallel to the dislocation line, such that a $360^{\circ}$ rotation about the line corresponds to a displacement of 1 Burgers vector in a smooth fashion. In the case of a pure edge dislocation in an isotropic material the solution is more complex, it is given by $\mathrm{Nabarro}^{4}$ for the case of the Burgers vector along $x$ as:

$$
\begin{aligned}
& u_{x}=\frac{b}{4 \pi(1-v)} \frac{x y}{\left(x^{2}+y^{2}\right)}-\frac{b}{2 \pi} \arctan \frac{x}{y}, \\
& u_{y}=-\frac{(1-2 v) b}{8 \pi(1-v)} \ln \frac{x^{2}+y^{2}}{b^{2}}+\frac{b}{4 \pi(1-v)} \frac{y^{2}}{\left(x^{2}+y^{2}\right)} \\
& u_{z}=0 .
\end{aligned}
$$

where $v$ is Poisson's ratio.

For eqns (A1) and (A2) to be valid the crystal must be elastically isotropic with only two independent elastic constants given by:

$$
\begin{aligned}
& s_{11}=s_{22}=s_{33} ; \\
& s_{12}=s_{13}=s_{23} ; \\
& s_{44}=s_{55}=s_{66}=2\left(s_{11}-s_{12}\right) .
\end{aligned}
$$

This means that, for almost all crystals, in order to find the correct displacement field needed to move an atom from its position in the perfect crystal into its location in the dislocated crystal, we must turn to the anisotropic elastic theory.

In the general case, with 21 independent elastic constants, analytical solutions are not readily available and the displacement field must be found numerically. ${ }^{42}$ However, several approaches have been suggested and symmetry simplifies the problem and several analytical solutions have been presented. ${ }^{43,47}$ In the simplest case, where the dislocation is straight, $z$ lies along a six-, four- or two-fold axis and another six-, four- or two-fold axis is perpendicular to it (chosen to lay along $x$ or $y$ ) the analytical solutions equivalent to eqns (1) and (2) are given by Steeds. ${ }^{43}$ (The presence of mirror planes can also lead to the same solutions for the anisotropic displacement field as a mirror plane can have the same effect on the elastic tensor as a rotation axis. The solution is valid if, using the axes described herein, the matrix representation of the elastic constant tensor reduces to the form:

$$
\begin{array}{cccccc}
c_{11} & c_{12} & c_{13} & 0 & 0 & 0 \\
& c_{22} & c_{23} & 0 & 0 & 0 \\
& & c_{33} & 0 & 0 & 0 \\
& & & c_{44} & 0 & 0 \\
& & & & c_{55} & 0 \\
& & & & & c_{66}
\end{array}
$$

or a more symmetrical form. Various combinations of fourand six-fold axes also have the effect of generating a matrix of this form. ${ }^{4,48}$ ) First, the elastic compliance matrix, $s_{l m}$, is converted into a reduced form, $S_{l m}$ (eqn. (A4)), in order to make use of the fact that the dislocation line is straight and there is no strain parallel to it:

$$
S_{l m}=s_{l m}-\frac{s_{3 l} S_{3 m}}{s_{33}}
$$


The expression for the linear elastic displacement field for a screw dislocation is then:

$$
\begin{aligned}
& u_{x}=0, \\
& u_{y}=0, \\
& u_{z}=\frac{b}{2 \pi} \arctan \left\{\sqrt{\left.\left(\frac{S_{44}}{S_{55}}\right) \frac{y}{x}\right\} .}\right.
\end{aligned}
$$

This expression reduces to the isotropic case (eqn. (A1)) when $S_{44}=S_{55}$. Otherwise, the effect is to change the pitch of the screw dislocation with the angle around the dislocation line.

For edge dislocations, the Burgers vector need not lie along one of the axes of the Cartesian frame of reference because the frame is chosen according to the symmetry of the crystal. First the components of the Burger's vector along $x$ and $y\left(b_{x}\right.$ and $\left.b_{y}\right)$ are calculated. Then, defining the anisotropic parameters:

$$
\begin{aligned}
\lambda^{4} & =\frac{S_{22}}{S_{11}}, \\
2 \Lambda & =-\frac{2 S_{12}+S_{66}}{S_{11}},
\end{aligned}
$$

the displacement field for each component can be found. For the $x$ component of the Burgers vector, with $\lambda^{4}<\Lambda^{2}$ (eqn. (A6)) the expressions for the atomic displacements are:

$$
\begin{aligned}
& u_{x}=\frac{b_{x}}{4 \pi}\left[\arctan \left\{\frac{\sqrt{2\left(\lambda^{2}-\Lambda\right)} \tan \theta}{1-\lambda^{2} \tan ^{2} \theta}\right\}-\frac{\left(\Lambda+S_{12} / S_{11}\right)}{\sqrt{\Lambda^{2}-\lambda^{4}}}\right. \\
& \left.\times \arctan \left\{\frac{\sqrt{-2\left(\Lambda+\lambda^{2}\right)} \tan \theta}{1+\lambda^{2} \tan ^{2} \theta}\right\}\right] \text {, } \\
& u_{y}=\frac{b_{x}}{4 \pi}\left[-\frac{\left(\lambda^{2}+S_{12} / S_{11}\right)}{\sqrt{2\left(\lambda^{2}-\Lambda\right)}} \ln r^{2} \sqrt{\cos ^{4} \theta+\lambda^{4} \sin ^{4} \theta-\frac{1}{2} \Lambda \sin ^{2} 2 \theta}\right. \\
& +\frac{\left(\lambda^{2}-S_{12} / S_{11}\right)}{2 \sqrt{-2\left(\Lambda+\lambda^{2}\right)}} \ln \left\{\frac{\cos ^{2} \theta-\left(\Lambda-\sqrt{\Lambda^{2}-\lambda^{4}}\right) \sin ^{2} \theta}{\cos ^{2} \theta-\left(\Lambda+\sqrt{\Lambda^{2}-\lambda^{4}}\right) \sin ^{2} \theta}\right\}, \\
& u_{z}=0
\end{aligned}
$$

the $y$ component of the Burger's vector gives:

$$
\begin{aligned}
& u_{x}=\frac{b_{y}}{4 \pi \lambda^{2}}\left[\frac{\left(\lambda^{2}+S_{12} / S_{11}\right)}{\sqrt{2\left(\lambda^{2}-\Lambda\right)}} \ln r^{2} \sqrt{\cos ^{4} \theta+\lambda^{4} \sin ^{4} \theta-\frac{1}{2} \Lambda \sin ^{2} 2 \theta}\right. \\
& +\frac{\left(\lambda^{2}-S_{12} / S_{11}\right)}{2 \sqrt{-2\left(\Lambda+\lambda^{2}\right)}} \ln \left\{\frac{\cos ^{2} \theta-\left(\Lambda-\sqrt{\Lambda^{2}-\lambda^{4}}\right) \sin ^{2} \theta}{\cos ^{2} \theta-\left(\Lambda+\sqrt{\Lambda^{2}-\lambda^{4}}\right) \sin ^{2} \theta}\right\}, \\
& u_{y}=\frac{b_{y}}{4 \pi}\left[\arctan \left\{\frac{\sqrt{2\left(\lambda^{2}-\Lambda\right)} \tan \theta}{1-\lambda^{2} \tan ^{2} \theta}\right\}+\frac{\left(\Lambda+S_{12} / S_{11}\right)}{\sqrt{\Lambda^{2}-\lambda^{4}}}\right. \\
& \left.\times \arctan \left\{\frac{\sqrt{-2\left(\Lambda+\lambda^{2}\right)} \tan \theta}{1+\lambda^{2} \tan ^{2} \theta}\right\}\right] \text {, }
\end{aligned}
$$

$u_{z}=0$.
In the case $\lambda^{4}>\Lambda^{2}$, the expressions for the $x$ components are:

$$
\begin{aligned}
u_{x}= & \frac{b_{x}}{4 \pi}\left[\arctan \left\{\frac{\sqrt{2\left(\lambda^{2}-\Lambda\right)} \tan \theta}{1-\lambda^{2} \tan ^{2} \theta}\right\}\right. \\
& +\frac{\left(\Lambda+S_{12} / S_{11}\right)}{\sqrt{\lambda^{4}-\Lambda^{2}}} \ln \left\{\frac{\cos ^{2} \theta+\lambda^{2} \sin ^{2} \theta-\sqrt{\frac{\Lambda+\lambda^{2}}{2}} \sin 2 \theta}{\cos ^{2} \theta+\lambda^{2} \sin ^{2} \theta+\sqrt{\frac{\Lambda+\lambda^{2}}{2}} \sin 2 \theta}\right\}, \\
u_{y}= & \frac{b_{x}}{4 \pi}\left[-\frac{\left(\lambda^{2}+S_{12} / S_{11}\right)}{\sqrt{2\left(\lambda^{2}-\Lambda\right)}} \ln r^{2} \sqrt{\cos ^{4} \theta+\lambda^{4} \sin ^{4} \theta-\frac{1}{2} \Lambda \sin ^{2} 2 \theta}\right. \\
& \left.+\frac{\left(\lambda^{2}-S_{12} / S_{11}\right)}{\sqrt{2\left(\Lambda+\lambda^{2}\right)}} \arctan \left\{\frac{\sqrt{\lambda^{4}-\Lambda^{2}}}{\cot ^{2} \theta-\Lambda}\right\}\right],
\end{aligned}
$$

$u_{z}=0$,

and the $y$ components become:

$$
\begin{aligned}
u_{x}= & \frac{b_{y}}{4 \pi \lambda^{2}}\left[-\frac{\left(\lambda^{2}+S_{12} / S_{11}\right)}{\sqrt{2\left(\lambda^{2}-\Lambda\right)}} \ln r^{2} \sqrt{\cos ^{4} \theta+\lambda^{4} \sin ^{4} \theta-\frac{1}{2} \Lambda \sin ^{2} 2 \theta}\right. \\
& \left.+\frac{\left(\lambda^{2}-S_{12} / S_{11}\right)}{\sqrt{2\left(\Lambda+\lambda^{2}\right)}} \arctan \left\{\frac{\sqrt{\lambda^{4}-\Lambda^{2}}}{\cot ^{2} \theta-\Lambda}\right\}\right], \\
u_{y}= & \frac{b_{y}}{4 \pi}\left[\arctan \left\{\frac{\sqrt{2\left(\lambda^{2}-\Lambda\right) \tan \theta}}{1-\lambda^{2} \tan ^{2} \theta}\right\}\right. \\
+ & \left.\frac{\left(\Lambda+S_{12} / S_{11}\right)}{\sqrt{\lambda^{4}-\Lambda^{2}}} \ln \left\{\frac{\cos ^{2} \theta+\lambda^{2} \sin ^{2} \theta-\sqrt{\frac{\Lambda+\lambda^{2}}{2}} \sin 2 \theta}{\cos ^{2} \theta+\lambda^{2} \sin ^{2} \theta+\sqrt{\frac{\Lambda+\lambda^{2}}{2}} \sin 2 \theta}\right\}\right],
\end{aligned}
$$

$u_{z}=0$.

Derivations of eqns. (A5)-(A10) can be found in Steeds. ${ }^{43}$ Analytical solutions for the displacement fields given by the anisotropic linear elastic theory are also available for the case of dislocation lines (1) lying along a three-fold axis with a perpendicular two-fold axis and (2) the case where the dislocation line is perpendicular to a two-fold axis. For systems of lower symmetry, numerical approaches are required to derive the elastic displacements.

Application of the above displacements to the atoms in the bulk-like cell result in the introduction of a dislocation. The atoms at a large distance from the dislocation line should be in the correct position but further calculation, utilising an atomistic model, is required to find the equilibrium structure of the dislocation core. Care must be taken in applying the elastic displacements, which must be calculated using the elastic constants that are generated by potential model, in addition the trigonometric functions must be used so as not to give discontinuities in the displacement field for angles of $\theta$ between $0^{\circ}$ and $360^{\circ}$. A. M. W. has developed a library of programs to enable the introduction of dislocations into model crystals, which are available upon correspondence.

\section{Acknowledgements}

K. W. thanks the Royal Society for support under their University Research Fellowship Scheme and J. D. G. gratefully 
acknowledges the support of the Government of Western Australia through a Premier's Research Fellowship. A. M. W. acknowledges a studentship from Engineering and Physical Sciences Research Council and thanks the Mineralogical Society for a Postgraduate travel bursary.

\section{References}

1 A. M. Walker, J. D. Gale, B. Slater and K. Wright, Phys. Chem. Chem. Phys., 2005, 7, DOI: 10.1039/b505716g.

2 J.-P. Poirier, Creep of Crystals. High-Temperature Deformation Processes in Metals Ceramics and Minerals, Cambridge University Press, Cambridge, 1985.

3 D. Hull and D. J. Bacon, Introduction to Dislocations, Pergamon Press, Oxford, 1984.

4 F. R. N. Nabarro, Theory of Dislocations, Oxford University Press, Oxford, 1967.

5 H. J. Frost and M. F. Ashby, Deformation Mechanism Maps. The Plasticity and Creep of Metals and Ceramics, Pergamon Press, Oxford, 1982, p. 166.

6 G. P. Bulanova, J. Geochem. Explor., 1995, 53, 1-23.

7 J. R. K. Bigger, D. A. McInnes, A. P. Sutton, P. M. C. I. Stich, R. D. King-Smith, D. M. Bird and L. J. Clarke, Phys. Rev. Lett., 1992, 69, 2224-2227.

8 W. Cai, V. V. Bulatov, J. Chang, J. Li and S. Yip, Phys. Rev. Lett., 2001, 86, 5727-5730.

9 S. Ismail-Beigi and T. A. Arias, Phys. Rev. Lett., 2000, 84, 14991502 .

10 F. Liu, M. Mostoller, M. F. Chisholm and T. Kaplan, Phys. Rev. $B, 1995, \mathbf{5 1}, 17192-17195$.

11 M. Mostoller, M. F. Chisholm and T. Kaplan, Phys. Rev. Lett., 1994, 72, 1494-1497.

12 M. I. Heggie, S. Jenkins, C. P. Ewels, P. Jemmer, R. Jones and P. R. Briddon, J. Phys.: Condens. Matter, 2000, 12, 10263-10270.

13 T. Kaplan, F. Liu, M. Mostoller, M. F. Chisholm and V. Milman, Phys. Rev. B, 2000, 61, 1674-1676.

14 (a) M. I. Heggie, C. P. Ewels, N. Martsinovich, S. Scarle, R. Jones, J. P. Goss, B. Hourahine and P. R. Briddon, J. Phys.: Condens. Matter, 2002, 14, 12689-12696; (b) N. Martsinovich, M. I. Heggie and C. P. Ewels, J. Phys.: Condens. Matter, 2003, 15, S2815S2824; (c) C. P. Ewels, N. T. Wilson, M. I. Heggie, R. Jones and P. R. Briddon, J. Phys.: Condens. Matter, 2001, 13, 8965-8972.

15 M. Leslie and M. J. Gillan, J. Phys. C: Solid State Phys., 1985, 18, 973-982.

16 J. E. Sinclair, P. C. Gehlen, R. G. Hoagland and J. P. Hirth, J. Appl. Phys., 1978, 49, 3890-3897.

17 J. E. Sinclair, J. Appl. Phys., 1971, 42, 5321-5329.

18 P. C. Gehlen, J. P. Hirth, R. G. Hoagland and M. F. Kanninen, J. Appl. Phys., 1972, 43, 3921-3933.

19 (a) L. H. Yang, P. Söderlind and J. A. Moriarty, Philos. Mag. A, 2001, 81, 1355-1385; (b) L. H. Yang, P. Söderlind and J. A. Moriarty, Mater. Sci. Eng., 2001, A309-310, 102-107.

20 S. Rao, D. Hernandez, J. P. Simmonds, T. A. Parthasarathy and C. Woodward, Philos. Mag. A, 1998, 77, 231-256.

21 (a) K. Ohsawa and E. Kuramoto, J. Appl. Phys., 1999, 86, 179-185; (b) Q.F. Fang and R. Wang, Phys. Rev. B, 2000, 62, 9317-9324.
22 W. Xu and J. A. Moriarty, Phys. Rev. B, 1996, 54, 6941-6951.

23 (a) J. H. Harding, Curr. Opin. Solid State Mater. Sci., 1997, 2 728-732; (b) V. V. Bulatov and L. P. Kubin, Curr. Opin. Solid State Mater. Sci., 1998, 3, 558-561; (c) J. Li, W. Cai, J. Chang and S. Yip, in Computational Materials Science, ed. C. R. A. Catlow and E. A. Kotomin, IOS Press, Amsterdam, 2003, pp. 359-387.

24 (a) T. Rasmussen, K. W. Jacobsen, T. Leffers and O. B. Pedersen, Phys. Rev. B, 1997, 52, 2977-2990; (b) T. Rasmussen, K. W. Jacobsen, T. Leffers, O. B. Pedersen, S. G. Srinivasan and H. Jónsson, Phys. Rev. Lett., 1997, 79, 3676-3679.

25 J. Marian, W. Cai and V. V. Bulatov, Nat. Mater., 2004, 3, 158-163.

26 M. P. Puls and M. J. Norgett, J. Appl. Phys., 1976, 47, 466-477.

27 R. G. Hoagland, J. P. Hirth and P. C. Gehlen, Philos. Mag., 1976, 34, 413-439.

28 C. H. Woo and M. P. Puls, J. Phys. C: Solid State Phys., 1976, 9, L27-L31.

29 C. H. Woo and M. P. Puls, Philos. Mag., 1977, 35, 727-756.

30 C. H. Woo and M. P. Puls, Philos. Mag., 1977, 35, 1641-1652.

31 (a) M. P. Puls, C. H. Woo and M. J. Norgett, Philos. Mag., 1977, 36, 1457-1472; (b) M. P. Puls, Philos. Mag. A, 1980, 41, 353-368; (c) M. P. Puls, Philos. Mag. A, 1983, 47, 497-513.

32 (a) J. Rabier and M. P. Puls, Philos. Mag. A, 1989, 59, 821-842; (b) J. Rabier, J. Soullard and M. P. Puls, Philos. Mag. A, 1990, 61, 99-108.

33 (a) J. H. Harding, Rep. Prog. Phys., 1990, 53, 1403-1466; (b) M. P Puls, in Dislocation Modelling of Physical Systems, ed. M. F. Ashby, R. Bullough, C. S. Hartley and J. P. Hirth, Pergamon Press, Oxford, 1981, pp. 249-268.

34 G. W. Watson, E. T. Kelsey, N. H. de Leeuw, D. J. Harris and S. C. Parker, J. Chem. Soc., Faraday Trans., 1996, 92, 433-438.

35 G. W. Watson, E. T. Kelsey and S. C. Parker, Philos. Mag. A, 1999, 79, 527-536.

36 G. W. Watson, P. M. Oliver and S. C. Parker, Surf. Sci. Lett., 2001, 474, L185-L190.

37 D. C. Sayle, J. Mater. Chem., 1999, 9, 607-616.

38 D. Wolf, P. Keblinski, S. R. Phillpot and J. Eggebrecht, J. Chem. Phys., 1999, 110, 8254-8282.

39 V. R. Saunders, C. Freyria-Fava, R. Dovesi and C. Roetti, Comput. Phys. Commun., 1994, 84, 156-172.

40 J. D. Gale and A. L. Rohl, Mol. Simul., 2003, 29, 291-341.

41 J. P. Hirth, Scr. Metall., 1972, 6, 535-540.

42 J. W. Steeds and J. R. Willis, in Dislocations in Solids, The Elastic Theory, ed. F. R. N. Nabarro, North-Holland Publishing Company, Amsterdam, 1979, pp. 143-165.

43 J. W. Steeds, Introduction to Anisotropic Elasticity Theory of Dislocations, Oxford University Press, Oxford, 1973.

44 J. S. Braithwaite, P. V. Sushko, K. Wright and C. R. A. Catlow, J. Chem. Phys., 2002, 116, 2628-2635.

45 A. M. Walker, B. Slater, J. D. Gale and K. Wright, Nat. Mater., 2004, 3, 715-720

46 B. K. D. Gairola, in Dislocations in Solids, The Elastic Theory, ed. F. R. N. Nabarro, North-Holland Publishing Company, Amsterdam, 1979, pp. 223-342.

47 J. P. Hirth and J. Lothe, Theory of Dislocations, John Wiley and Sons, New York, 1982.

48 J. F. Nye, Physical Properties of Crystals. Their Representation by Tensors and Matrices, Clarendon press, Oxford, 1957. 